\title{
Long-term outcome of myotomy and fundoplication based on intraoperative real-time high-resolution manometry in achalasia patients
}

\author{
Tania Triantafyllou ${ }^{a}$, Charalampos Theodoropoulos ${ }^{\mathrm{a}}$, Georgia Georgioua, Vasileios Kalles ${ }^{\mathrm{a}}$, \\ Demosthenis Chrysikos ${ }^{b}$, Konstantinos Filis ${ }^{a}$, Georgios Zografos ${ }^{a}$, Dimitrios Theodorou ${ }^{a}$ \\ Hippocration General Hospital of Athens, University of Athens; General and Oncologic Hospital of Kifissia Agii \\ Anargiri, Athens, Greece
}

\section{Abstract}

\begin{abstract}
Background Current treatment options for achalasia of the esophagus predominantly consist of endoscopic myotomy or laparoscopic myotomy combined with a partial fundoplication. The intraoperative use of conventional manometry has previously been proposed with various results. The aim of the present study was to introduce the use of high-resolution manometry (HRM) during surgical treatment for achalasia and to assess the long-term outcome of this technique.
\end{abstract}

Methods We enrolled achalasia patients within the time period November 2013 to July 2016 who underwent HRM and evaluation of Eckardt scores (ES) before and after tailored laparoscopic myotomy and fundoplication with intraoperative recording using HRM.

Results Twenty patients were classified as having achalasia type I (20\%), type II (55\%), or type III (25\%). During surgery, 9 myotomies were extended and 13 fundoplications were modified according to HRM findings. Mean resting (16.1 vs. $41.9 \mathrm{mmHg}$ ) and residual (9 vs. $28.7 \mathrm{mmHg}$ ) pressures of the lower esophageal sphincter and ES (0.7 vs. 6.9) were significantly eliminated postoperatively over a mean follow-up time of 17.7 months.

Conclusions The use of intraoperative HRM gives us the advantage of simultaneous real-time estimation of intraluminal pressures of the esophagus and the ability to identify the exact points that produce pressure during laparoscopy. Consequently, it may be the key to the tailoring of the Heller-Dor technique and improving the outcomes for achalasia patients.

Keywords Esophageal achalasia, high-resolution manometry, fundoplication

Ann Gastroenterol 2019; 32 (1): 1-6

Department of Surgery, ${ }^{\mathrm{a}} \mathrm{s}^{\text {st }}$ Propaedeutic Surgical Clinic, Hippocration General Hospital of Athens, University of Athens (Tania Triantafyllou, Charalampos Theodoropoulos, Georgia Georgiou, Vasileios Kalles, Konstantinos Filis, Georgios Zografos, Dimitrios Theodorou); ${ }^{\mathrm{b}}$ General and Oncologic Hospital of Kifissia "Agii Anargiri"

(Demosthenis Chrysikos), Athens, Greece

Conflict of Interest: None

Correspondence to: Tania Triantafyllou, MD, PhD, Foregut Surgical Department, Hippocration General Hospital of Athens, University of Athens, Greece, 114 Vas. Sofias Ave., 11527 Athens, Greece,

e-mail: t_triantafilou@yahoo.com

Received 19 July 2018; accepted 16 October 2018;

published online 14 November 2018

DOI: https://doi.org/10.20524/aog.2018.0326

\section{Introduction}

Achalasia of the esophagus is a primary motility disorder characterized by impaired peristalsis of the esophageal body and incomplete relaxation of the lower esophageal sphincter (LES). The etiology of the disease still remains unclear. The incidence is 1.6 cases per 100,000 individuals per year. Highresolution manometry (HRM) of the esophagus is the goldstandard diagnostic tool for achalasia, as it is a precise and evident technology that classifies the disorder into subtypes according to the Chicago Classification [1,2]. The 3 subtypes are classic achalasia (type I), achalasia with panesophageal pressurization (type II) and vigorous achalasia (type III), based on the pattern of motility of the esophageal body. The severity of symptoms is most commonly evaluated using the Eckardt score (ES). The ES is the most commonly used clinical scoring 
system in achalasia, as it tends to be the most useful scale in clinical practice. It takes into consideration the frequency and severity of dysphagia, regurgitation and chest pain in addition to weight loss evaluation on a scale of $0-12$. Each symptom is graded from 0-3 [3].

Since medical treatment for achalasia has shown poor results, endoscopic and surgical procedures are predominantly applied worldwide. All therapeutic approaches are considered palliative, as esophageal motility in achalasia cannot be restored [4]. Laparoscopic myotomy followed by a partial fundoplication, also known as the Heller-Dor procedure, is from the long-term aspect the most effective treatment, with a $90 \%$ success rate [5]. Surgical failure is mainly attributed to either incomplete myotomy or tight fundoplication, and most frequently presents as postoperative dysphagia [6].

With regard to improving the surgical outcome, the intraoperative use of manometry was proposed in a few studies several years ago with varying results. All studies published used conventional manometry, mainly comparing instantaneous values of the intraluminal pressures of the esophagus before and after myotomy and fundoplication. The main aim was to calibrate the dynamic effect of every surgical step and in some cases modify the technique accordingly [7-14]. The wide use of HRM, however, has eliminated the use of conventional manometry in current practice, as the new generation manometry is undoubtedly a more accurate tool for the evaluation of esophageal motility. The main objective of this study was to evaluate both clinically and functionally the long-term results of modifications of the standardized surgical technique based on simultaneous and continuous manometric recording using HRM.

\section{Patients and methods}

In this prospective study we enrolled consecutive patients diagnosed with achalasia based on upper gastrointestinal tract endoscopy and HRM. All participants were adults with no previous history of surgery in the upper gastrointestinal tract.

Based on manometric findings and the Chicago Classification (CC), the patients were classified into achalasia subtypes. A detailed history and the severity of achalasia symptoms using the ES were recorded preoperatively. Patient demographics were also reviewed. All individuals signed an informed consent document in order to participate in the study. The ethics committee of our institution approved the study protocol.

The population studied underwent laparoscopic myotomy and fundoplication by a single surgeon, using the HRM probe during the procedure in order to calibrate the manometric profile of all surgical steps during the surgical technique, as described previously by our department [15]. Modifications of either myotomy or fundoplication based on increased pressure zones during the Heller-Dor procedure were also calibrated. All patients were reassessed with ES and a repeat manometric test with HRM after surgery.

The HRM studies were performed with a ManoScan ESO Given Imaging catheter that consists of 36 pressure channels.
Data acquisition, display and analysis were performed with ManoView Software, Given Imaging. The manometric tests consisted of ten 5-mL swallows in the supine position. The reports were evaluated with the current version of CC v3.0.

The participants enrolled to this protocol underwent laparoscopic myotomy and an individually modified partial fundoplication according to the intraoperative manometric values. HRM was used to calibrate the entire surgical procedure, while the patient was under general anesthesia. In fact, the real-time recording started at the time of intubation and the manometric analysis was simultaneously assessed on the HRM screen, placed next to the laparoscopic monitor, in such a way that each surgical step (retraction, mobilization, myotomy, fundoplication) could be evaluated manometrically. The setup of the HRM in the operating room has been thoroughly described in our previous study [15]. Although the standardized Heller myotomy is a $5-\mathrm{cm}$ esophagomyotomy from the esophagogastric junction (EGJ) proximally to the esophagus and $2 \mathrm{~cm}$ distally to the cardia of the stomach, in this study the total length of myotomy to the esophagus or distally to the stomach was adapted to the pressures recorded during surgery. More precisely, residual high pressures along the EGJ were eliminated by further cutting the circular muscularis propria of the esophagus or incising any remaining circumferential esophageal vessels after localization of the exact anatomic point of the residual high-pressure zone using the forceps (Fig. 1). In addition, if high pressures caused by the standard sutures required it, Dor fundoplication was modified by skipping the right side of the myotomized esophagus, fixing the right side of the myotomy to the right crus or adding more sutures to minimize tension to the lower esophagus (Fig. 2).

Statistical analysis was conducted using the Statistical Package for the Social Sciences (IBM, SPSS, version 23.0). For normally distributed data, a paired-samples $t$-test was conducted to evaluate the impact of our intervention on patient values. To inquire about possible correlations between

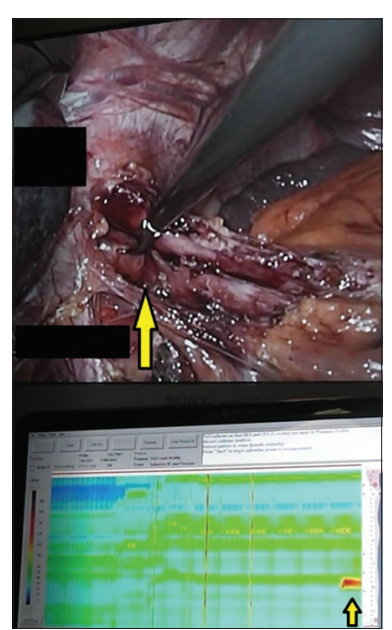

Figure 1 Identification of incomplete myotomy The surgeon uses a laparoscopic forceps (big yellow arrow) to identify the high-pressure zone recorded by high-resolution manometry (small yellow arrow) 


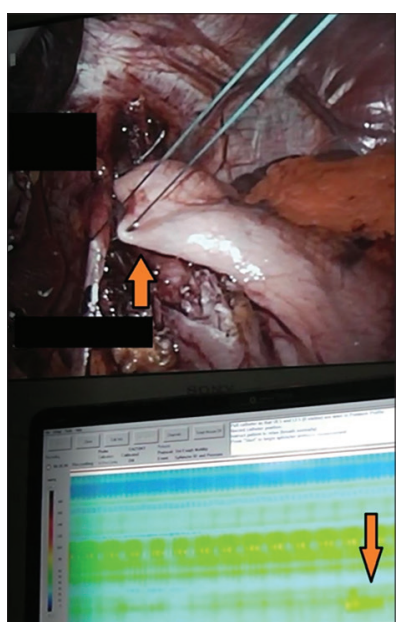

Figure 2 Identification of tight fundoplication

The surgeon identifies an increase in the pressure of the gastroesophageal junction (orange arrow in the high-resolution manometry screen) to identify the high-pressure zone caused by the last suture of the fundoplication (orange arrow in the laparoscopic screen)

patient values, the Pearson correlation coefficient was used. For all statistical tests, a P-value $<0.05$ was considered significant.

\section{Results}

Twenty-four achalasia patients were enrolled in our study between September 2013 and July 2016. All volunteers were diagnosed with achalasia of the esophagus based on HRM findings. All individuals underwent HRM before surgery and completed the ES. However, only 20 patients eventually repeated the postoperative manometric study with HRM. Of these, 20\% were diagnosed with achalasia type I, 55\% with type II, and $25 \%$ with type III, according to the CC v3.0. The mean age at the time of diagnosis was $50.8 \pm 14.7$ (17-83) years and $45 \%$ of the patients were female.

Regarding the surgical technique, Heller myotomy was further extended in 9 cases (45\% of patients) according to manometric findings during the procedure. Because of elevated pressures according to the HRM, Dor fundoplication was modified in thirteen patients (65\%), either by the addition of an extra suture between the gastric fundus and the central tendon of the diaphragm (45\%), or by relocation of one of the sutures (5\%) that comprised the wrap. We also applied a modified technique in 3 patients (15\%), concerning the last two sutures of the standardized Dor fundoplication, which consisted of fixation of the gastric fundus directly to the right crus of the diaphragm, as previously described [16]. In addition, in 7 patients (35\%) we performed incision of the short gastric vessels because high pressures persisted after completion of the wrap. Figure 3 presents the aforementioned modifications.

The procedures were completed with no intraoperative complications and all patients were discharged on the second postoperative day. Liquid diet was initiated on the first postoperative day. The mean postoperative follow-up time was

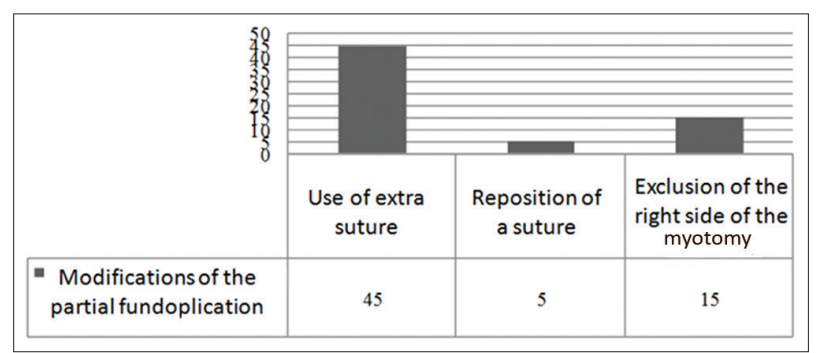

Figure 3 Modifications of the Dor technique based on intraoperative high-resolution manometry findings (\%)

17.7 (range 7-48) months. Regarding the mean pressures of the LES, both resting and residual pressures significantly decreased after surgery (16.16 vs. $41.97 \mathrm{mmHg}, \mathrm{P}<0.001$, and 9 vs. $28.78 \mathrm{mmHg}, \mathrm{P}<0.001$, respectively) (Table 1 ). In fact, these findings were significant regardless of the achalasia subtype (Table 2). Among the HRM parameters of the esophageal body, distal contractile integral (DCI) was the only parameter that significantly decreased after surgery (1382.64 vs. $3745.24 \mathrm{mmHg} \cdot \mathrm{sec} \cdot \mathrm{cm}, \mathrm{P}=0.05$ ) (Table 3). A statistically significant decrease was also found regarding the ES after surgery (0.74 vs. $6.91, \mathrm{P}<0.001)$.

\section{Discussion}

Over the years, pharmacotherapy and endoscopic techniques (injection of botulinum toxin and dilations) had predominantly been established as the standard of care regarding treatment for achalasia of the esophagus. As the long-term efficiency of these options has been questioned, current advances in the treatment of achalasia mainly focus on the outcome of the standard surgical Heller-Dor procedure and the evolving technique of peroral endoscopic myotomy (POEM), widely performed. Although the current literature still lacks long-term results, the short-term results of POEM were initially promising; however, recent studies have revealed increasing rates of reflux [17]. Moreover, according to a study by Patel et al, endoscopy after POEM resulted in a new diagnosis of esophagitis in $19 \%$ of the patients who underwent the procedure [17]. In fact, a multicenter study concluded that more than $55 \%$ of patients presented with a DeMeester score of $\geq 14.72$ after a median follow up of one year [18].

Taking under consideration the limitations of POEM, improving the result of surgical myotomy combined with fundoplication may be a reasonable approach in order to achieve the optimal outcome. Calibration and individualization of the technique based on the intraoperative manometric findings may be the key to a further reduction in the rates of postoperative failure. In the past, several studies proposed the use of manometry during surgical treatment for achalasia, aiming to calibrate and, in some cases, guide myotomy and fundoplication. However, there is no study that applied HRM intraoperatively. Case series, case reports and short communications have been published, including adult and pediatric patients diagnosed with achalasia [7-14]. 
Table 1 HRM characteristics of the LES before and after surgery

\begin{tabular}{|c|c|c|c|c|}
\hline Characteristic & Mean & SD & SE & P-value \\
\hline \multicolumn{5}{|c|}{ LES resting pressure } \\
\hline Preoperatively & 41.97 & & & \\
\hline Postoperatively & 16.16 & 12.43 & 2.85 & $<0.001$ \\
\hline \multicolumn{5}{|c|}{ LES residual pressure } \\
\hline Preoperatively & 28.78 & & & \\
\hline Postoperatively & 9.00 & 12.08 & 2.77 & $<0.001$ \\
\hline \multicolumn{5}{|l|}{ Pressures in $\mathrm{mmHg}$} \\
\hline \multicolumn{5}{|c|}{ A P-value $<0.05$ was considered statistically significant } \\
\hline
\end{tabular}

More precisely, Mattioli et al studied the role of intraoperative manometry during esophagogastric myotomy and achieved abolition of the high-pressure zone in all patients, according to intraoperative manometric measurements. Moreover, the great majority of the patients presented with improvement of their symptoms after surgery [7]. A few more studies presented their results regarding recession of the symptoms of patients who underwent myotomy and fundoplication with intraoperative conventional manometry [8-14].

The present study evaluated the long-term outcomes of our previously presented pilot study on the intraoperative use of HRM during surgical treatment for achalasia of the esophagus [15]. HRM provides the advantage of simultaneous and continuous recording of the pressures of the esophageal lumen during all surgical steps of the Heller-Dor technique. Most importantly, it may guide further myotomy or modification of the fundoplication in cases where a high residual pressure is recorded. At the same time, the surgeon is able to localize the exact point in the esophagus by using a grasper over the high-pressure zone in order to identify the anatomic point presenting with functional abnormality.

Regarding modifications based on our protocol, 9 myotomies ( $45 \%$ of patients) were further extended after completion of the standardized extent of the Heller technique, while 13 cases $(65 \%)$ underwent a modified partial technique as a result of increased residual pressures during the established Dor fundoplication. Additionally, in 7 patients (35\%) the short gastric vessels were sacrificed in order to achieve a floppier wrap. Clinical and objective reassessment of the patients with ES and HRM tests, respectively, after surgery revealed a significant decrease in total ES (0.5 vs. 7.5$)$, resting (16.16 vs. $41.97 \mathrm{mmHg}$ ) and residual (9 vs. $28.78 \mathrm{mmHg}$ ) pressures of the LES, along with the DCI measurements (1382.6 vs. $3745.2 \mathrm{mmHg} \cdot \mathrm{sec} \cdot \mathrm{cm}$ ). Although postoperative HRM findings in surgical patients are rarely reported in the literature, the study of Salvador et al revealed a decrease in median resting pressure of the LES (15.3 vs. $39 \mathrm{mmHg}$ ) and residual pressure of the LES (6 vs. $27.4 \mathrm{mmHg}$ ) [19] The decrease in our DCI measurements to one third of the preoperative value may also indicate the adequacy of resolution of the previously recorded high pressure of the LES, diminished after the myotomy. Moreover, the effect of the partial fundoplication on esophageal body contraction did not increase the DCI values postoperatively. The value of the changes in the pattern of esophageal body contraction after endoscopic or surgical treatment among the 3 types of achalasia has been already discussed in the literature; however, the clinical significance of the difference between preoperative and postoperative HRM measurements, such as DCI or esophageal peristaltic amplitude, still remains unclear. Interestingly, the possibility of partial recovery of the esophageal body motility after POEM or surgical myotomy cannot be ruled out $[20,21]$.

Furthermore, given the fact that surgical intervention for achalasia aims to improve the patient's quality of life, the most important outcome of our study was the decrease in postoperative ES compared to the preoperative evaluation (0.7 vs. 6.9), which represents patients' satisfaction regarding dysphagia, regurgitation, chest pain and weight gain after surgery. In a study by Gockel et al, the ES decreased from 6 to 1 for 108 patients who underwent myotomy along with Dor fundoplication for achalasia of the esophagus [22]. In the present study, postoperative symptoms were almost diminished during the long-term follow-up period.

Our study included a small number of patients; however, this is the first reference in the literature to assess the long-term outcomes of the application of HRM during surgical treatment for achalasia of the esophagus. Although the EGJ contractile integral is currently the most meaningful HRM metric in the evaluation of EGJ function, this study was initiated before the first introduction of this measurement [23]. Therefore, it was the residual and resting pressures of the LES that were assessed in our HRM studies. Another limitation may be the difficulty in defining precise cutoff values for the decision to modify the technique. Objective values could not be used, as general anesthesia and pneumoperitoneum established at a pressure of $12 \mathrm{mmHg}$ interfere with the manometric values of the EGJ during surgery; thus, preoperative HRM assessment could not be directly related to intraoperative pressures. To address this problem, we intraoperatively compared the pressures of the EGJ to the esophageal body pressures or the pressure recorded in the stomach. Another drawback of the present study may be the lack of objective evaluation of postoperative reflux. Postoperative reflux was assessed using the ES and the need for proton pump inhibitors. Although locating the previous LES position in order to place a $\mathrm{pH}$ probe or capsule may be confusing, because of the significant decrease in pressure, future studies may elucidate the value of $\mathrm{pH}$ tests postoperatively. Overall, regardless of the demanding setup of the HRM software and hardware and acquisition of measurements in the theater, the application of the HRM during the Heller-Dor technique may be a useful and safe guide, especially for the new surgeon, as it provides a detailed recording of the functional result of each surgical step.

The technique of impedance planimetry (endoluminal functional lumen imaging probe; endoFLIP) has been applied during both surgical and endoscopic treatment of achalasia, aiming to guide the length of myotomy and fundoplication [24,25]. However, the idea of instant measurements of diameter and distensibility of the esophagus during myotomy with the endoFLIP seems to differ from the 
Table 2 HRM characteristics of the LES before and after surgery according to achalasia subtype

\begin{tabular}{|c|c|c|c|c|c|c|c|c|c|}
\hline \multirow[t]{2}{*}{ Characteristic } & \multicolumn{3}{|c|}{ Achalasia type I $(n=4)$} & \multicolumn{3}{|c|}{ Achalasia type II $(n=11)$} & \multicolumn{3}{|c|}{ Achalasia type III $(n=5)$} \\
\hline & Mean & $\mathrm{SD}$ & P-value & Mean & SD & P-value & Mean & SD & P-value \\
\hline \multicolumn{10}{|c|}{ LES resting pressure } \\
\hline Preoperatively & 40.86 & & & 37.57 & & & 51.00 & & \\
\hline Postoperatively & 7.02 & 5.85 & $<0.001$ & 18.59 & 14.48 & 0.004 & 20.94 & 5.77 & $<0.001$ \\
\hline \multicolumn{10}{|c|}{ LES residual pressure } \\
\hline Preoperatively & 26.66 & \multirow{2}{*}{6.7} & \multirow{2}{*}{0.002} & 25.30 & \multirow{2}{*}{11.63} & \multirow{2}{*}{0.002} & 40.13 & \multirow{2}{*}{17.49} & \multirow{2}{*}{0.058} \\
\hline Postoperatively & 4.80 & & & 9.10 & & & 14.00 & & \\
\hline
\end{tabular}

Pressures in $\mathrm{mm}$ Hg, Length in $\mathrm{cm}$; P-value $<0.05$ was considered statistically significant

HRM, high-resolution manometry; LES, lower esophageal sphincter; SD, standard deviation

Table 3 HRM parameters of the body of the esophagus

\begin{tabular}{|c|c|c|c|c|}
\hline Parameters & Mean & SD & SE & P-value \\
\hline \multicolumn{5}{|l|}{ DCI } \\
\hline Preoperatively & 3745.24 & \multirow{2}{*}{3244.18} & \multirow{2}{*}{1025.9} & \multirow{2}{*}{0.05} \\
\hline Postoperatively & 1382.64 & & & \\
\hline \multicolumn{5}{|l|}{ CFV } \\
\hline Preoperatively & 8139.15 & \multirow{2}{*}{25857.96} & \multirow{2}{*}{8177.01} & \multirow{2}{*}{0.35} \\
\hline Postoperatively & 54.81 & & & \\
\hline \multicolumn{5}{|l|}{ DL } \\
\hline Preoperatively & 3.46 & \multirow{2}{*}{5.58} & \multirow{2}{*}{1.77} & \multirow{2}{*}{0.87} \\
\hline Postoperatively & 3.75 & & & \\
\hline \multicolumn{5}{|l|}{ Velocity } \\
\hline Preoperatively & 22.64 & \multirow{2}{*}{42.67} & \multirow{2}{*}{13.49} & \multirow{2}{*}{0.5} \\
\hline Postoperatively & 13.20 & & & \\
\hline \multicolumn{5}{|l|}{ \%Failed Swallows } \\
\hline Preoperatively & 43.33 & \multirow{2}{*}{49.19} & \multirow{2}{*}{11.59} & \multirow{2}{*}{0.06} \\
\hline Postoperatively & 66.94 & & & \\
\hline \multicolumn{5}{|c|}{ P-value $<0.05$ was considered statistically significant } \\
\hline $\begin{array}{l}\text { HRM, high-resolution } \\
\text { mmHg.sec.cm); CFV, } \\
\text { sec); pressures in mm } \\
\text { rror }\end{array}$ & $\begin{array}{l}\text { nometry; } D \\
\text { tractile fror }\end{array}$ & $\begin{array}{l}\text { I, distal con } \\
\text { velocity (cm } \\
; \text { SD, standa }\end{array}$ & $\begin{array}{l}\text { ctile integ } \\
\left.c^{-1}\right) ; D L, d \\
\text { deviation }\end{array}$ & $\begin{array}{l}\text { atency } \\
\text { tandard }\end{array}$ \\
\hline
\end{tabular}

intraoperative use of HRM, as our protocol presents realtime continuous manometric recording of all surgical steps, precisely synchronized to the laparoscopic camera view in a more dynamic and detailed way.

This study is the first presentation of the long-term results of the use of HRM during surgical treatment for achalasia of the esophagus. Based on intraoperative manometric findings, we further extended $45 \%$ of the myotomies and we modified $65 \%$ of the fundoplications in the group of patients we enrolled. The results of the present study proved that the intraoperative use of HRM may be a useful tool for safely tailoring myotomy and fundoplication in order to achieve the optimal functional and clinical outcome. Future case-control or even randomized studies with a larger number of patients may be mandatory.

\section{Summary Box}

\section{What is already known:}

- Laparoscopic Heller myotomy combined with Dor fundoplication for the treatment of achalasia of the esophagus achieves a $90 \%$ success rate

- Failure of the surgical technique is attributed to either incomplete myotomy or tight fundoplication

- Previous studies manometrically calibrated the surgical effect of the myotomy and fundoplication during the surgical procedure using conventional manometry with various results

- High-resolution manometry (HRM) is the goldstandard tool for the assessment of esophageal motility

\section{What the new findings are:}

- This study is the first presentation of the long-term clinical and manometric results of the use of HRM during surgical treatment for achalasia of the esophagus

- The results of the present study proved that intraoperative HRM may be a useful tool for safely tailoring the myotomy and fundoplication to achieve the optimal functional and clinical outcome

\section{References}

1. Kahrilas PJ, Bredenoord AJ, Fox M, et al; International High Resolution Manometry Working Group. The Chicago Classification of esophageal motility disorders, v3.0. Neurogastroenterol Motil 2015;27:160-174.

2. Kahrilas PJ, Bredenoord AJ, Fox M, et al; International Working Group for Disorders of Gastrointestinal Motility and Function. Expert consensus document: Advances in the management of oesophageal motility disorders in the era of high-resolution manometry: a focus on 
achalasia syndromes. Nat Rev Gastroenterol Hepatol 2017;14:677-688.

3. Gockel I, Junginger T. The value of scoring achalasia: a comparison of current systems and the impact on treatment-the surgeon's viewpoint. Am Surg 2007;73:327-331.

4. Vaezi MF, Pandolfino JE, Vela MF. ACG clinical guideline: diagnosis and management of achalasia. Am J Gastroenterol 2013;108:12381249; quiz 1250.

5. Campos GM, Vittinghoff E, Rabl C, et al. Endoscopic and surgical treatments for achalasia: a systematic review and meta-analysis. Ann Surg 2009;249:45-57.

6. Patti MG, Allaix ME. Recurrent symptoms after Heller myotomy for achalasia: evaluation and treatment. World J Surg 2015;39:1625-1630.

7. Mattioli S, Pilotti V, Felice V, Di Simone MP, D’Ovidio F, Gozzetti G. Intraoperative study on the relationship between the lower esophageal sphincter pressure and the muscular components of the gastro-esophageal junction in achalasic patients. Ann Surg 1993;218:635-639.

8. Di Martino N, Monaco L, Izzo G, et al. The effect of esophageal myotomy and myectomy on the lower esophageal sphincter pressure profile: intraoperative computerized manometry study. Dis Esophagus 2005;18:160-165.

9. Endo S, Nakajima K, Nishikawa K, et al. Laparoscopic Heller-Dor surgery for esophageal achalasia: impact of intraoperative realtime manometric feedback on postoperative outcomes. Dig Surg 2009;26:342-348.

10. Corcione F, Cristinzio G, Cimmino V, et al. Surgical laparoscopy with intraoperative manometry in the treatment of esophageal achalasia. Surg Laparosc Endosc 1997;7:232-235.

11. Jafri $\mathrm{M}$, Alonso $\mathrm{M}$, Kaul A, et al. Intraoperative manometry during laparoscopic Heller myotomy improves outcome in pediatric achalasia. J Pediatr Surg 2008;43:66-70.

12. Khan M, Smythe A, Elghellal K, et al. Can intraoperative manometry during laparoscopic fundoplication predict postoperative dysphagia? Surg Endosc 2010;24:2268-2272.

13. Clemente G, D'Ugo D, Granone P, Nuzzo G, Picciocchi A. Intraoperative esophageal manometry in surgical treatment of achalasia: a reappraisal. Hepatogastroenterology 1996;43:1532-1536.

14. Chapman JR, Joehl RJ, Murayama KM, et al. Achalasia treatment: improved outcome of laparoscopic myotomy with operative manometry. Arch Surg 2004;139:508-513.

15. Triantafyllou T, Doulami G, Papailiou J, Mantides A, Zografos G, Theodorou D. Real-time continuous esophageal high-resolution manometry (HRM) during laparoscopic Heller myotomy and Dor fundoplication for the treatment of achalasia. A promising novelty in regards of perfecting surgical technique: could It guide surgical technique toward excellent results? Surg Laparosc Endosc Percutan Tech 2016;26:e163-e166.

16. Triantafyllou T, Natoudi M, Ntanasis-Stathopoulos I, et al. Dor fundoplication for achalasia of the esophagus: is it time to simplify the established technique? evaluation of clinical outcome. Int Surg J 2017;4:2615-2619.

17. Patel K, Abbassi-Ghadi N, Markar S, Kumar S, Jethwa P, Zaninotto G. Peroral endoscopic myotomy for the treatment of esophageal achalasia: systematic review and pooled analysis. Dis Esophagus 2016;29:807-819.

18. Kumbhari V, Familiari P, Bjerregaard NC, et al. Gastroesophageal reflux after peroral endoscopic myotomy: a multicenter casecontrol study. Endoscopy 2017;49:634-642.

19. Salvador R, Savarino E, Pesenti E, et al. The impact of Heller myotomy on integrated relaxation pressure in esophageal achalasia. J Gastrointest Surg 2016;20:125-131; discussion 131.

20. Roman S, Kahrilas PJ, Mion F, et al. Partial recovery of peristalsis after myotomy for achalasia: more the rule than the exception. JAMA Surg 2013;148:157-164.

21. Hu Y, Li M, Lu B, Meng L, Fan Y, Bao H. Esophageal motility after peroral endoscopic myotomy for achalasia. J Gastroenterol 2016;51:458-464.

22. Gockel I, Junginger T, Eckardt VF. Long-term results of conventional myotomy in patients with achalasia: a prospective 20-year analysis. J Gastrointest Surg 2006;10:1400-1408.

23. Gor P, Li Y, Munigala S, Patel A, Bolkhir A, Gyawali CP. Interrogation of esophagogastric junction barrier function using the esophagogastric junction contractile integral: an observational cohort study. Dis Esophagus 2016;29:820-828.

24. Ilczyszyn A, Hamaoui K, Cartwright J, Botha A. Intraoperative distensibility measurement during laparoscopic Heller's myotomy for achalasia may reduce the myotomy length without compromising patient outcome. Dis Esophagus 2016;29:455-462.

25. Ngamruengphong $\mathrm{S}$, von Rahden $\mathrm{BH}$, Filser J, et al. Intraoperative measurement of esophagogastric junction cross-sectional area by impedance planimetry correlates with clinical outcomes of peroral endoscopic myotomy for achalasia: a multicenter study. Surg Endosc 2016;30:2886-2894. 